\title{
Improvement of Phase Synchronization between Chaotic Oscillators with External Force in a Laboratory Plasma
}

\author{
Takao Fukuyama, Kengo Hagimine, and Ren Miyazaki \\ Faculty of Education, Nagasaki University, \\ 1-14 Bunkyo-machi, Nagasaki 852-8521, Japan
}

(Received May 7, 2017)

\begin{abstract}
The improvement of phase synchronization between two coupled chaotic oscillators achieved using a pulse as the external force in a glow discharge plasma is experimentally demonstrated. The effect of coupling and pulse application on the dynamic behaviors of systems is investigated. From the viewpoint of the phase of oscillations, experimental results show that phase synchronization between two coupled chaotic oscillators is improved by applying a pulse.
\end{abstract}

Plasma is considered an ideal medium for investigating the properties of nonlinear phenomena. Consequently, a whole range of studies on chaos in plasma have been conducted, such as the observation of chaos, ${ }^{1,2)}$ control of chaos, ${ }^{3,4)}$ and synchronization of chaos. ${ }^{5,6)}$ The various stages of the phenomenon of chaos synchronization, such as complete synchronization, ${ }^{7,8)}$ lag synchronization, ${ }^{9,10)}$ and phase synchronization, ${ }^{11,12)}$ have been investigated theoretically and experimentally. Chaos synchronization in coupled nonlinear oscillators $^{13)}$ as well as chaotic behaviors has been observed in a laboratory plasma. ${ }^{5,6,14,15)}$ In complete synchronization, amplitudes and phases are completely synchronized between two oscillators. On the other hand, in phase synchronization, only phases are synchronized between two oscillators. Because of the sensitivity of coupled chaotic oscillators toward external perturbation, there is a possibility that applying an external force changes the phase correlation of coupled systems from disorder to order. In this note, experimental findings on the improvements of phase synchronization between two coupled chaotic oscillators achieved by using an external force in a laboratory plasma are reported.

The schematic of the experimental setup is shown in Fig. 1. Experiments are performed using two glass tubes with a diameter and length of $0.02 \mathrm{~m}$ and $0.75 \mathrm{~m}$, respectively. Neon gas is confined in the two tubes at a pressure of approximately $478 \mathrm{~Pa}$ after evacuating each tube to a high vacuum. When a high de potential is applied to the electrodes, Ne plasma is produced as a glow discharge between the electrodes. A resistor with a resistance of $9.4 \mathrm{k} \Omega$ 
is incorporated in the circuit of tube 1 in order to sustain the discharge. The transformer incorporated in the circuit of tube 2 has a resistance of $8.0 \mathrm{k} \Omega$. Time series signals for the analysis are obtained as fluctuations in the light intensity of Ne plasma using photodiodes (S6775, HAMAMATSU) and are sampled using a digital oscilloscope (GDS-1072A-U, GWINSTEK). The photodiodes on tubes 1 and 2 are placed at a distance of $0.17 \mathrm{~m}$ from the anode. High dc voltages are generated using a regulated dc power source (TMK 1.0-50, TAKASAGO for discharge in tube 1 and HV1.5-0.3, TAKASAGO for discharge in tube 2). The external force, which is a pulse in this study, is generated using a function generator (33220A, AGILENT). The pulse is amplified using an amplifier (4015, NF ELECTRONIC INSTRUMENTS) and superimposed using a transformer (EF-4N, SHIMADZU) incorporated in the circuit of tube 2 . Ionization waves ${ }^{16)}$ are self-excited and unstable owing to the ionized instability in Ne plasma produced as a glow discharge. ${ }^{15,17-20)}$ Typical electron and ion temperatures in plasma are $10 \mathrm{eV}$ and $0.025 \mathrm{eV}$, respectively. The discharge currents in tubes 1 and 2 are changed to govern the states of the systems. Waves 1 and 2 are sampled from tubes 1 and 2, respectively. Two nonlinear waves interact with each other through electric coupling. "Before coupling" refers to the state in which two waves do not interact, and "after coupling" refers to the state in which two waves interact. The ionization waves exhibit a wide variety of oscillations such as periodic and chaotic oscillations when the values of the discharge current $I$ and gas pressure are changed. In our experiment, the gas pressure is fixed at $478 \mathrm{~Pa}$. When the discharge currents in tubes 1 and 2 are fixed at $29.0 \mathrm{~mA}$ and $19.0 \mathrm{~mA}$, respectively, each wave has a broad spectrum that exhibits chaotic oscillations. A pulse (square wave) provided as an external force has a fundamental frequency of $1.1 \mathrm{kHz}$. The intensity of the pulse is comparable with the electric potential and sustains discharge in tubes 1 and 2. A pulse has all the components of sine waves. Therefore, it works effectively as an external force to perturb systems compared to waves of other shapes.

The effect of coupling and pulse application on the dynamic behaviors of systems is investigated. The Lyapunov exponents are calculated to perform chaotic analysis to examine the effect quantitatively. The largest Lyapunov exponents are calculated from the time series of waves 1 and 2 sampled from the photodiodes on tubes 1 and 2, based on the algorithm reported in reference ${ }^{21)}$ and by using a time series obtained in a string of experiments. The value of the largest Lyapunov exponent is positive for chaotic oscillations; this value is higher for a more chaotic system. The value becomes close to zero for a system with periodic oscillations. Table 1 lists the values of the largest Lyapunov exponents of waves 1 and 2, and their total value. With coupling, the value increases from 0.380 to 0.474 when no pulse is 
applied, and it increases from 0.375 to 0.463 on application of the pulse. Therefore, the system attains a more chaotic state on coupling. With pulse application, the value changes from 0.380 to 0.375 before coupling, and it changes from 0.474 to 0.463 after coupling. Therefore, the turbulence of systems is not largely altered on applying a pulse. From the results of calculating the largest Lyapunov exponents, it is found that coupling and pulse application do not work to suppress the amplifier turbulence of systems.

We shift the perspective to focus from the amplitude to phase of oscillations. Figure 2 shows the time evolution of the phase difference $\left|\Phi_{2}-\Phi_{1}\right|$ between waves 1 and 2 . It shows $\left|\Phi_{2}-\Phi_{1}\right|$ in the case of $\diamond$, which represents the state before coupling and before applying the pulse; $\mathbf{O}$, which represents the state after coupling and before applying the pulse; $\triangle$, which represents the state before coupling and after applying the pulse; and $\times$, which represents the state after coupling and after applying the pulse. The phase difference $\left|\Phi_{2}-\Phi_{1}\right|$ is calculated using the following method. One period is defined as the time from a certain maximum value to the next maximum of the amplitude in a time series, following which only maximum value points are extracted. The times corresponding to the maximum values of waves 1 and 2 are compared in series, following which the phase difference $\left|\Phi_{2}-\Phi_{1}\right|$ is calculated from these results. From the viewpoint of coupling, the slope of phase differences between waves 1 and 2 becomes more gradual owing to the coupling in both cases without and with the application of the pulse.

Furthermore, when the discharge currents in both tubes 1 and 2 are set to the same value, the time evolutions of the phase difference in the cases after coupling and before applying the pulse as well as after coupling and after applying the pulse are compared in order to examine the effect of applying the pulse on phase synchronization. Two tubes are electrically connected in series, and the fundamental frequency of pules is fixed at $2.3 \mathrm{kHz}$. Figure $3(\mathrm{a})$ shows the relationship between the value of discharge current and the slope of the phase difference between waves 1 and $2, \Delta\left|\Phi_{2}-\Phi_{1}\right| / \Delta t$. The slope of the phase difference is calculated by the least squares method. Figure 3(b) shows the relationship between the value of discharge current and the largest Lyapunov exponents. represents the state after coupling and before applying the pulse, and $\times$ represents the state after coupling and after applying the pulse. The error bars are calculated using the standard deviation. The values of slopes $(-$ and $\times$ ) approach each other with increasing discharge current. The results of calculating the largest Lyapunov exponents show that pulse application does not work to 
suppress the amplifier turbulence of systems. It is shown that the phases of coupled systems are stabilized by applying a pulse when the discharge current is greater than $26 \mathrm{~mA}$. Therefore, experimental results demonstrate that phase synchronization between two chaotic oscillators is improved when an appropriate discharge current is selected and a pulse is used as an external force.

The findings are summarized as follows. We investigated the improvement of phase synchronization between two coupled chaotic oscillators achieved using an external force in a glow discharge plasma. The effect of coupling and pulse application on the dynamic behaviors of systems was investigated. The Lyapunov exponents were calculated to perform chaotic analysis. The results indicated that coupling and pulse application failed to suppress the amplifier turbulence of systems. Next, the focus was shifted from the amplitude to the phase of oscillations. From the results, it was observed experimentally that phase synchronization between the two chaotic oscillators improved when using a pulse as an external force. 
1) P. Y. Cheung, S. Donovan, and A. Y. Wong, Phys. Rev. Lett. 61, 1360 (1988).

2) M. Ohno, M. Tanaka, A. Komori, and Y. Kawai, J. Phys. Soc. Jpn. 58, 28 (1989).

3) Th. Pierre, G. Bonhomme, and A. Atipo, Phys. Rev. Lett. 76, 2290 (1996).

4) K. Taniguchi and Y. Kawai, Phys. Rev. Lett. 83, 548 (1999).

5) T. Fukuyama and Y. Kawai, J. Phys. Soc. Jpn. 71, 1809 (2002).

6) P. V. Popov, R. A. Filatov, A. A. Koronovskii, and A. E. Hramov, Tech. Phys. Lett. 31, 221 (2005).

7) H. Fujisaka and T. Yamada, Prog. Theor. Phys. 69, 32 (1983).

8) L. M. Pecora and T. L. Carroll, Phys. Rev. Lett. 64, 821 (1990).

9) M. G. Rosenblum, A. S. Pikovsky, and J. Kurths, Phys. Rev. Lett. 78, 4193 (1997).

10) S. Taherion and Y. Lai, Phys. Rev. E 59, R6247(R) (1999).

11) M. G. Rosenblum, A. S. Pikovsky, and J. Kurths, Phys. Rev. Lett. 76, 1804 (1996).

12) E. Rosa, Jr., E. Ott, and M. H. Hess, Phys. Rev. Lett. 80, 1642 (1998).

13) A. T. Winfree, J. Theor. Biol. 16, 15 (1967).

14) M. Agop, P. Nica, O. Niculescu, and D.G. Dimitriu, J. Phys. Soc. Jpn. 81, 064502 (2012).

15) N. Chaubey, S. Mukherjee, A. N. Sekar Iyengar, and A. Sen, Phys. Plasmas 22, 022312 (2015).

16) M. Novak, Czech. J. Phys. 10, 954 (1960).

17) Md. Nurujjaman, R. Narayanan, and A. N. Sekar Iyengar, Chaos 17, 043121 (2007).

18) N. L. Oleson and A. W. Cooper, Adv. Electron El. Phys. 24, 155 (1968).

19) M. Rottmann and K. H. Spatschek, J. Plasma Phys. 60, 215 (1998).

20) T. Fukuyama and M. Okugawa, Phys. Plasmas 24, 032302 (2017).

21) A. Wolf, J. B. Swift, H. L. Swinney, and J. A. Vastano, Physica 16D, 285 (1985). 
Table 1. Values of the largest Lyapunov exponents $\lambda_{\max }$ of waves 1 and 2 , as well as their total value. Here, $\lambda 1_{\max }$ denotes the $\lambda_{\max }$ of wave 1 and $\lambda 2_{\max }$ denotes the $\lambda_{\max }$ of wave 2.

\begin{tabular}{|c|c|c|c|c|}
\hline Coupling & Pulse application & $\lambda 1_{\max }$ & $\lambda 2_{\max }$ & $\lambda 1_{\max }+\lambda 2_{\max }$ \\
\hline before & before & 0.213 & 0.166 & 0.380 \\
\hline after & before & 0.251 & 0.223 & 0.474 \\
\hline before & after & 0.199 & 0.176 & 0.375 \\
\hline after & after & 0.250 & 0.213 & 0.463 \\
\hline
\end{tabular}

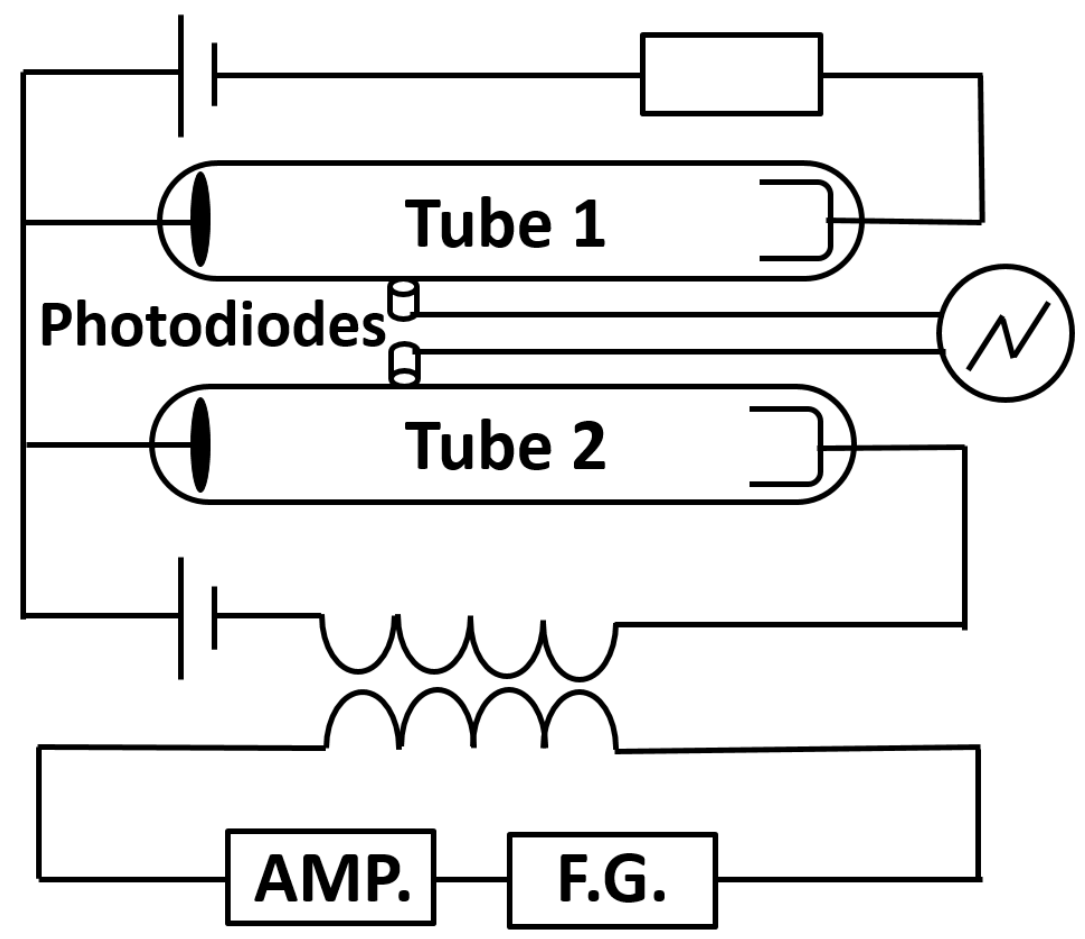

Fig. 1. Schematic configuration of the experimental setup. 




Fig. 2. Time evolution of the phase difference $\left|\Phi_{2}-\Phi_{1}\right|$ between waves 1 and $2 . \diamond$ represents the state before coupling and before applying the pulse,

represents the state after coupling and before applying the pulse, $\triangle$ represents the state before coupling and after applying the pulse, and $\times$ represents the state after coupling and after applying the pulse. 


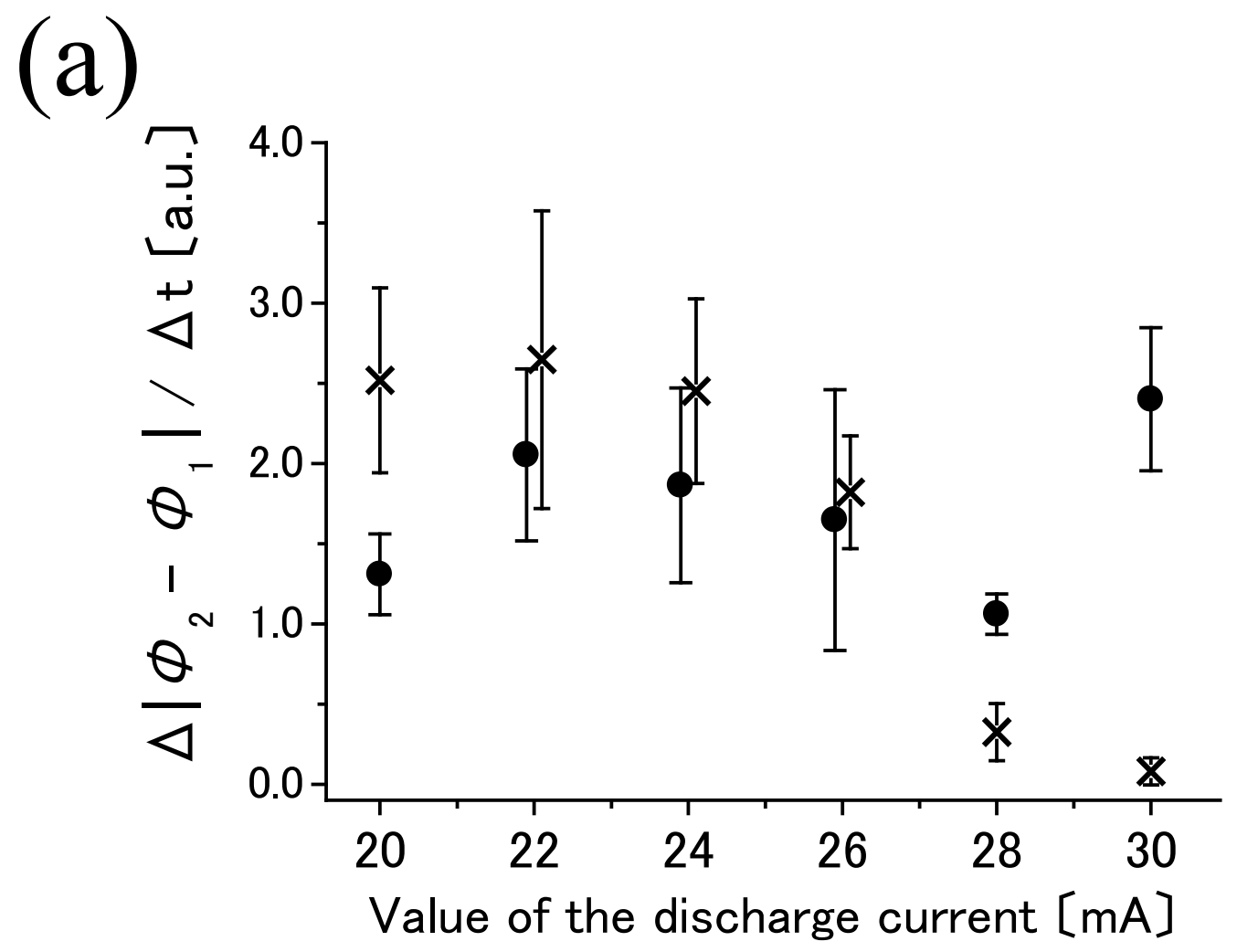

(b)

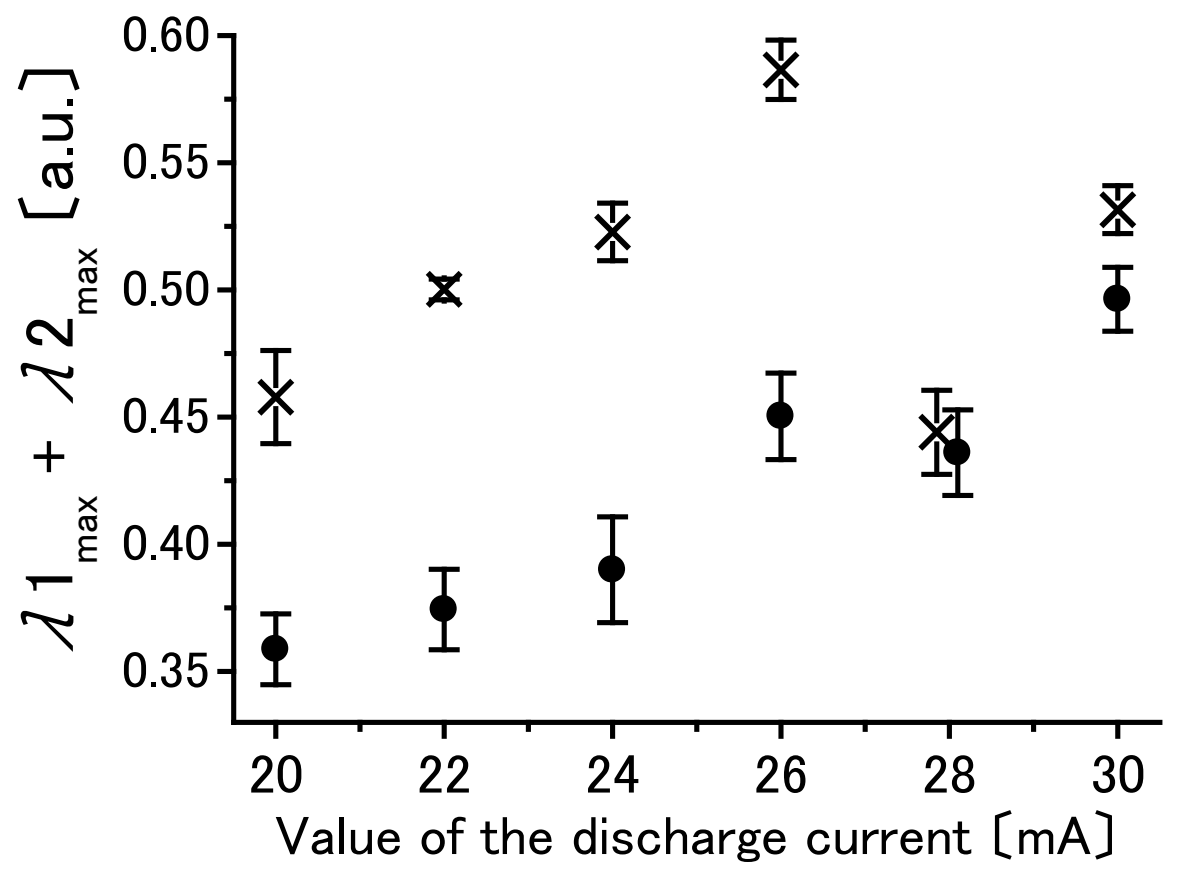

Fig. 3. (a) Relationship between the value of discharge current and the slope of the phase difference between waves 1 and 2; (b) relationship between the value of discharge current and the largest Lyapunov exponents. represents the state after coupling and before applying the pulse, and $\times$ represents the state after coupling and after applying the pulse. 